\title{
Inhibition de la corrosion d'un acier au carbone par des produits dérivés de phosphonates en association avec des sels de zinc
}

\section{Corrosion inhibition of a carbon steel by the association of phosphonate-derived products with zinc salts}

\author{
M. C. Lafont, N. Pebere, F. Moran et P. Bleriot
}

Volume 6, numéro 1, 1993

URI : https://id.erudit.org/iderudit/705168ar

DOI : https://doi.org/10.7202/705168ar

\section{Aller au sommaire du numéro}

\section{Éditeur(s)}

Université du Québec - INRS-Eau, Terre et Environnement (INRS-ETE)

ISSN

0992-7158 (imprimé)

1718-8598 (numérique)

Découvrir la revue

Citer cet article

Lafont, M. C., Pebere, N., Moran, F. \& Bleriot, P. (1993). Inhibition de la corrosion d'un acier au carbone par des produits dérivés de phosphonates en association avec des sels de zinc. Revue des sciences de l'eau / Journal of Water Science, 6(1), 97-112. https://doi.org/10.7202/705168ar

\section{Résumé de l'article}

Dans cette étude, des mesures électrochimiques ont été utilisées pour caractériser l'efficacité inhibitrice de produits dérivés de phosphonates associés à des sels de zinc, employés pour le traitement des eaux des circuits de refroidissement. L'influence de la concentration de cette formulation ainsi que l'effet du pH du milieu ont été étudiés.

Les courbes courant-tension stationnaires et les diagrammes d'impédance électrochimique ont été obtenus avec des électrodes tournantes en acier au carbone dans une solution de chlorure de sodium à $200 \mathrm{mg} \mathrm{l}^{-1}$. Ce milieu a été choisi car sa faible conductivité électrique est proche de celle rencontrée dans les eaux naturelles.

La méthode stationnaire (relevé des courbes courant-tension) a permis de déterminer la vitesse de corrosion en l'absence et en présence de l'inhibiteur et par conséquent, le taux de protection. Ainsi, le composé présente une très bonne efficacité dès les faibles concentrations $\left(50 \mathrm{mg} \cdot \mathrm{l}^{-1}\right)$. Entre 50 et $200 \mathrm{mg}$. $\mathrm{l}^{-1}$, l'efficacité inhibitrice augmente de 95 à $98 \%$. Pour la concentration de 100 $\mathrm{mg} \cdot \mathrm{l}^{-1}$ il est efficace dans un large domaine de $\mathrm{pH}$ (de 5,5 à 9). Cette efficacité apparaît légèrement supérieure à $\mathrm{pH}=7$ et à $\mathrm{pH}=8$.

Les valeurs des résistances de polarisation mesurées à partir des diagrammes d'impédance confirment les résultats obtenus à partir des courbes stationnaires.

En outre, l'efficacité inhibitrice du composé a été comparée à celle de produits déjà testés au Laboratoire pour des utilisations identiques. Le chlorure de zinc, le monofluorophosphate de zinc et l'association d'une amine grasse et d'acide phosphonique présentent des efficacités moindres que le mélange testé ici à base de produits dérivés de phosphonates associés aux sels de zinc. 


\title{
Inhibition de la corrosion d'un acier au carbone par des produits dérivés de phosphonates en association avec des sels de zinc
}

\author{
Corrosion inhibition of a carbon steel by the association \\ of phosphonate-derived products with zinc salts
}

M.C. LAFONT1, N. PEBERE1, F. MORAN2, P. BLERIOT2

Reçu le 25 févier 1992 , accepté pour publication le 21 septembre 1992*.

In this study, electrochemical measurements were carried out to characterize the inhibitive efficiency of the association of phosphonate-derived products with zinc salts, employed for water treatment in cooling circuits.

The influence of parameters such as the concentration of the formulation and the effect of the solution's pH on the protection of the metal were studied.

Steady-state current-voltage curves and electrochemical impedance diagrams have been obtained for a carbon steel rotating disc in a $200 \mathrm{mg} \cdot \mathrm{I}^{-1} \mathrm{NaCl}$ solution. The choice of this medium is based upon the following criteria :

(i) its low electrical conductivity is close to that encountered in natural saline waters;

(ii) its corrosivity is fairly high;

(iii) it is formed by an easily reproducible baseline solution.

The inhibitive efficiency has been determined from the plotting of the cathodic potentiostatic curves. It is important to note that in low conductivity media, the current-voltage curves are distorted by a non-negligible uncompensated ohmic drop (l. $\left.\mathbf{R}_{0}\right), \mathbf{R}_{8}$ being the electrolyte resistance between the working and the reference electrodes. The interpretation of these curves (i.e. corrosion rate evaluation, Tafel slopes determination) is only possible if they have been corrected according to the following relationship.

$$
E_{\text {apparent }}=E_{\text {true }}+R_{6} \mathbf{I}
$$

In this study, the electrolytic resistance value has been determined from electrochemical impedance measurements : the high frequency limit of the impedance gives $\mathbf{R}_{\mathbf{e}}$.

1. URA CNRS 445, Equipe de Métallurgie Physique - E.N.S.C.T. - , 118 Route de Narbonne, 31077 Toulouse cedex, France. Téléptone : 61.17 .56 .65 .

2. Concorde Chimie France, 145, Rue de Belleville, 75019 Paris. Téléphone : 16 (1) 42.40.33.76.

* Les commentaires seront rę̧us jusqu'au 14 août 1993. 
The ohmic drop is then automatically compensated by the potentiostat to obtain the corrected steady-state curve.

The inhibitive efficiency is evaluated by the relationship :

$$
E_{i}=\frac{i_{\text {corr }}^{0}-I_{\text {cor }}}{i_{\text {cor }}^{0}} \times 100
$$

in which $\mathrm{i}^{0}$ corr and $\mathrm{i}_{\text {eorr }}$ represent the current densities without and with inhibitor, respectively.

In addition to the determination of the current densities obtained from the steady-state current-voltage curves, the polarization resistance, $\mathbf{R}_{p}$, has been measured from the impedance diagram : the low frequency limit of the impedance gives the sum $R_{e}+R_{p} ; R_{p}$ is equal to the slope of the steady-state cur rent voltage curve.

In this study, only the evolution of this value as a function of concentration has been examined.

It has been shown from the steady-state technique that the compound presents a very good efficiency for low concentrations $\left(50 \mathrm{mg} \cdot \mathrm{r}^{-1}\right)$. Between 50 and 200 $\mathrm{mg} \cdot \mathrm{F}^{-1}$, the inhibitive efficiency increases from 95 to $98 \%$. For $100 \mathrm{mg} \cdot \mathrm{I}^{-1}$ it is effective over a wide $\mathrm{pH}$ range $(5.5$ to 9$)$ with a better protection for $\mathrm{pH}=7$ and $\mathrm{pH}=8$.

The measurements of the polarization resistance corroborate these results.

In a second part, the inhibitive efficiency of the compound has been compared to different inhibitors used for similar uses and tested in the laboratory. Thus, zinc chloride, zinc fluorophosphate and the association of fatty amine and phosphonic acid present a lower efficiency than the product tested here.

Key-words : Inhibition, corrosion rate, electrochemical methods, water treatment, zinc salts, phosphonates.

Dans cette étude, des mesures électrochimiques ont été utilisées. pour caractériser l'efficacité inhibitrice de produits dérivés de phosphonates associés a des sels de zinc, employés pour le traitement des eaux des circuits de refroidissement. L'influence de la concentration de cette formulation ainsi que l'effet du pH du milieu ont été ćtudiés.

Les courbes courant-tension stationnaires et les diagrammes d'impédance électrochimique ont été obtenus avec des électrodes tournantes en acier au carbone dans une solution de chlorure de sodium a $200 \mathrm{mg} \cdot \mathrm{I}^{-1}$. Ce milieu a été choisi car sa faible conductivité électrique est proche de celle rencontrée dans les eaux naturelles.

La méthode stationnaire (relevé des courbes courant-tension) a permis de déterminer la vitesse de corrosion en l'absence et en présence de l'inhibiteur et par conséquent, le taux de protection. Ainsi, le composé présente une très bonne efficacité dès les faibles concentrations $\left(50 \mathrm{mg} \cdot \mathrm{l}^{-1}\right)$. Entre 50 et $200 \mathrm{mg} \cdot \mathbf{1}^{-1}$, l'efficacité inhibitrice augmente de 95 a $98 \%$. Pour la concentration de $100 \mathrm{mg} \cdot \mathrm{I}^{-1}$ il est efficace dans un large domaine de $\mathrm{pH}$ (de 5,5 à 9). Cette efticacité apparaît légèrement supérieure à $\mathrm{pH}=7$ et à $\mathrm{pH}=8$.

Les valeurs des résistances de polarisation mesurées à partir des diagrammes d'impédance confirment les résultats obtenus à partir des courbes stationnaires. 
En outre, l'efficacité inhibitrice du composé a été comparée à celle de produits déjà testés au Laboratoire pour des utilisations identiques. Le chlorure de zinc, le monofluorophosphate de zinc et l'association d'une amine grasse et d'acide phosphonique présentent des efficacités moindres que le mélange testé ici a base de produits dérivés de phosphonates associés aux sels de zinc.

Mots clés : Inhibition, vitesse de corrosion, méthodes électrochimiques, traitement de l'eau, sels de zinc, phosphonates.

\section{1 - INTRODUCTION}

Parmi les inhibiteurs de corrosion généralement utilisés pour le traitement des eaux industrielles figurent de nombreuses substances minérales telles que les chromates, les nitrites, les sulfites, les phosphates, les polyphosphates. Cependant, leur utilisation est limitée soit pour des raisons techniques, telle que la température d'hydrolyse pour les polyphosphates, soit pour des raisons de toxicité, notamment en ce qui concerne les chromates et les nitrites. Devant cet état de fait, la recherche de nouveaux inhibiteurs de corrosion s'imposait.

Dans cette étude, une nouvelle formulation à base de produits dérivés de phosphonates et de polyacrylates associés à des sels de zinc a été testée. Le choix de cette composition est justifié par les propriétés de ces produits qui agissent a la fois comme inhibiteur de corrosion et comme inhibiteur d'entartrage dans les circuits de refroidissement.

Nous avons entrepris de caractériser l'efficacité inhibitrice du composé à l'aide de méthodes électrochimiques stationnaire (tracé des courbes de polarisation) et transitoire (mesure de limpédance électrochimique).

Le milieu corrosif est une solution à $200 \mathrm{mg} \cdot \mathrm{F}^{-1} \mathrm{de}$ chlorure de sodium. Ce milieu a été choisi car sa faible conductivité électrique est proche de celle rencontrée dans les eaux naturelles (DUPRAT, LAFONT, MORAN et ROCHER, 1985).

Nous présentons ici les résultats relatifs, d'une part à l'influence de la concentration de cette formulation et, d'autre part, à l'effet du pH sur la protection du matériau. De plus, nous comparons l'efficacité inhibitrice du composé à celle de produits déjà testés au Laboratoire pour des utilisations identiques. 


\section{2 - CONDITIONS EXPÉRIMENTALES}

\subsection{Matériau et milieu}

L'acier retenu dans cette étude est un acier au carbone XC 35 (Norme AFNOR). Le milieu corrosif est constitué par une solution à $200 \mathrm{mg} \cdot \mathrm{l}^{-1} \mathrm{de}$ chlorure de sodium RP normapur de marque Prolabo dans de l'eau permutée. Tous les essais ont été effectués à température ambiante.

Le pH de la solution à $200 \mathrm{mg} \cdot \mathrm{I}^{-1}$ de $\mathrm{NaCl}$ est de 5,5 . II a été ajusté avec de la soude pour obtenir les valeurs de 7,8 et 9 .

\subsection{Mesures électrochimiques}

Les essais électrochimiques ont été menés à l'aide d'une électrode à disque tournant de surface $1 \mathrm{~cm}^{2}$; la vitesse de rotation est fixée à 1000 tr. $\mathrm{mn}^{-1}$.

Le dispositif expérimental permettant le tracé des courbes courant-tension et des diagrammes d'impédance est un montage classique à trois électrodes (électrode de travail, contre-électrode en platine, électrode de référence au calomel saturé (E.C.S.)) reliées à un ensemble Schlumberger Solartron composé :

- d'une interface électrochimique Schlumberger 1286 permettant le contrôle de la polarisation, la correction automatique de chute ohmique indispensable lors des essais dans le milieu peu conducteur, les balayages potentiostatiques automatiques (un incrément de $20 \mathrm{mV}$ est appliqué toutes les deux minutes) et un affichage complet des caractéristiques mesurées,

- d'un analyseur de réponse en fréquence Schlumberger 1250 qui opère un balayage automatique de la fréquence lors des essais d'impédance électrochimique (domaine de fréquences : $63 \mathrm{kHz}$ à quelques millihertz avec 8 points par décade).

L'ensemble est piloté par un ordinateur (HP 9000 série 300).

\subsection{Protocole opératoire des essais électrochimiques}

L'électrode en acier XC 35 est polie au papier émeri de grade 80 , nettoyée dans l'eau déminéralisée sous ultrasons et séchée. L'élèctrode est ensuite plongée dans la solution agressive.

Après 2 heures d'immersion au potentiel de corrosion, on effectue une mesure d'impédance électrochimique (la durée de l'essai est de 1 heure). Cette mesure n'affectant pas le système (les signaux utilisés sont de faible amplitude $(.10 \mathrm{mV})$ ), la courbe de polarisation est ensuite tracée à partir du potentiel de corrosion vers des potentiels de plus en plus cathodiques.

L'état de surface de l'électrode est observé à la fin de ces essais soit environ après 5 heures d'immersion.

Les courbes, diagrammes et tableaux présentés sont le reflet d'au moins deux manipulations. 


\section{3-RÉSULTATS EXPÉRIMENTAUX ET DISCUSSION}

L'efficacité inhibitrice a été déterminée à l'aide d'une méthode électrochimique basée sur l'exploitation des courbes courant-tension stationnaires relevées en mode potentiostatique dans le domaine cathodique.

L'efficacité inhibitrice, $E_{i}$, est alors évaluée par la relation :

$$
\dot{E_{i}}=\frac{i_{\text {corr }}^{\circ}-i_{\text {corr }}}{i_{\text {corr }}^{\circ}} \times 100
$$

dans laquelle $i_{\text {corr }}$ et $i_{\text {corr }}$ représentent les densités de courant de corrosion en présence et en l'absence de l'inhibiteur, respectivement.

D'après une analyse critique des processus électrochimiques de la corrosion d'un acier au carbone en milieux neutres chlorurés décrite en détail par DUPRAT, LAFONT, MORAN et ROCHER (1985), les densités de courant de corrosion ont été déterminées par extrapolation au potentiel de corrosion de la droite de TAFEL. Cette dernière, du fait de l'influence de la diffusion, ne peut être mise en évidence qu'après correction de la courbe $I=f(E)$ suivant la relation $1 / /=1 / I_{k}+1 / I_{L}$ dans laquelle $I_{k}$ représente la densité de courant correspondant au seul transfert de charge, I la densité de courant mixte et $I_{L}$ la densité de courant limite de diffusion.

II est important de noter qu'en milieu faiblement conducteur, les courbes courant-tension $(I, E)$ seront déformées par la présence d'une chute ohmique non compensée. L'exploitation de ces courbes ne pourra être effectuée qu'à partir des courbes corrigées selon la relation:

$$
E_{\text {Vrai }}=E_{l u}-R_{\theta} \mid
$$

dans laquelle $E_{l u}$ représente le potentiel imposé, $R_{\theta}$ la résistance de l'électrolyte et l le courant résultant de la polarisation.

Dans cette étude, la valeur de la résistance électrolytique a été mesurée à partir des diagrammes d'impédance électrochimique : la valeur de $R_{e}$ est la limite réelle de l'impédance lorsque la fréquence tend vers l'infini. Elle est de l'ordre de $600 \Omega \cdot \mathrm{cm}^{2}$. La valeur de la résistance électrolytique ainsi mesurée est fournie à l'interface électrochimique qui applique une correction sur la réaction positive du potentiostat, permettant ainsi d'obtenir directement les courbes courant-tension corrigées de la chute ohmique.

A la détermination de la densité de courant de corrosion obtenue à partir du tracé des courbes de polarisation stationnaires, nous avons ajouté la mesure de la résistance de polarisation, $R_{p}$, obtenue à l'aide de l'exploitation des diagrammes d'impédance (la valeur de la résistance de polarisation est la limite réelle de limpédance quand $\omega \rightarrow 0$ ).

Compte-tenu de la déformation importante que présentent les diagrammes d'impédance et de la difficulté d'extrapolation de la partie basse fréquence dans certains cas, nous nous sommes limités à examiner l'évolution de la résistance de polarisation en fonction de la concentration. 


\subsection{Influence de la concentration en inhibiteur}

\subsubsection{Courbes courant-tension stationnaires}

\section{a) Dans le domaine cathodique}

La concentration en inhibiteur recommandée par la Société Concorde Chimie France est de $100 \mathrm{mg} \cdot \mathrm{r}^{-1}$. L'action inhibitrice du composé a été étudiée en outre pour deux concęntrations inférieures et deux concentrations supérieures à celle préconisée.

L'ensemble des résultats obtenus est regroupé dans le (tableau 1) alors que les (fig. 1 et 2) représentent les courbes courant-tension correspondantes.

A partir de ces résultats, nous observons que :

1 - Quelle que soit la concentration en composé (fig. 1), l'allure des courbes au voisinage du potentiel de corrosion, $E_{\text {Corr }}$, fait apparaître un large domaine de cinétique mixte (activation-diffusion) avec un plateau de diffusion de plus en plus étroit lorsque la concentration en inhibiteur augmente. $\mathrm{Ce}$ plateau tend à disparaître dès que l'on atteint la concentration de $150 \mathrm{mg} \cdot \mathrm{1}^{-1}$.

Il est important de noter que, pour des valeurs de potentiel cathodique (de

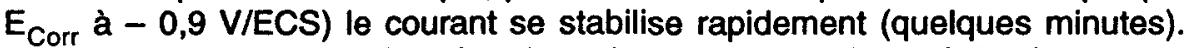
Ainsi, dans le domaine étudié l'adsorption du composé paraît indépendante du potentiel validant de ce fait la correction de diffusion sur la courbe couranttension.

2 - Pour la concentration la plus faible $(25 \mathrm{mg} / \mathrm{l})$ la hauteur du plateau de diffusion est supérieure à celle obtenue sans inhibiteur. Ce paradoxe apparent peut s'expliquer par le fait que, dans la solution non inhibée, la corrosion du métal conduit à la formation de couches poreuses de produits de corrosion qui recouvrent la surface. Celles-ci limitent la réaction de réduction de l'oxygène et abaissent fortement le plateau cathodique. La valeur du courant limite mesurée est alors nettement plus faible que le courant calculé par Levich sur une surface uniformément réactive. En présence d'inhibiteur, les produits de corrosion se forment en quantité moins importante et de ce fait, la surface de l'électrode devient plus accessible pour la réaction cathodique. Conjointement, on note un déplacement du potentiel de corrosion vers des potentiels plus positifs. Dans ce cas, l'inhibiteur n'apporte qu'une protection partielle de la surface. La valeur de l'efficacité mesurée est de $80 \%$ (tableau 1).

3 - Lorsque la concentration en inhibiteur augmente, l'efficacité inhibitrice augmente. Ainsi, dès $50 \mathrm{mg} \cdot 1^{-1}$, la protection apportée par l'inhibiteur est satisfaisante et la surface de l'électrode apparaît intacte après les essais de corrosion. Entre 50 et $200 \mathrm{mg} \cdot \mathrm{1}^{-1}$, l'efficacité inhibitrice mesurée passe de 95 à $98 \%$. La valeur du potentiel de corrosion $\left(-414 \mathrm{mV} / \mathrm{ECS}\right.$ pour $\left.25 \mathrm{mg} \cdot \mathrm{1}^{-1}\right)$ se déplace vers des potentiels de plus en plus cathodiques lorsque la concentration augmente, jusqu'à atteindre la valeur de $E_{\text {corr }}$ mesurée dans le cas de la solution non inhibée. Ce comportement associé à l'abaissement du plateau de diffusion peut s'expliquer par une action cathodique de l'inhibiteur, due, principalement à la présence de sels de zinc dans le mélange inhibiteur, qui se renforcerait avec la concentration. 

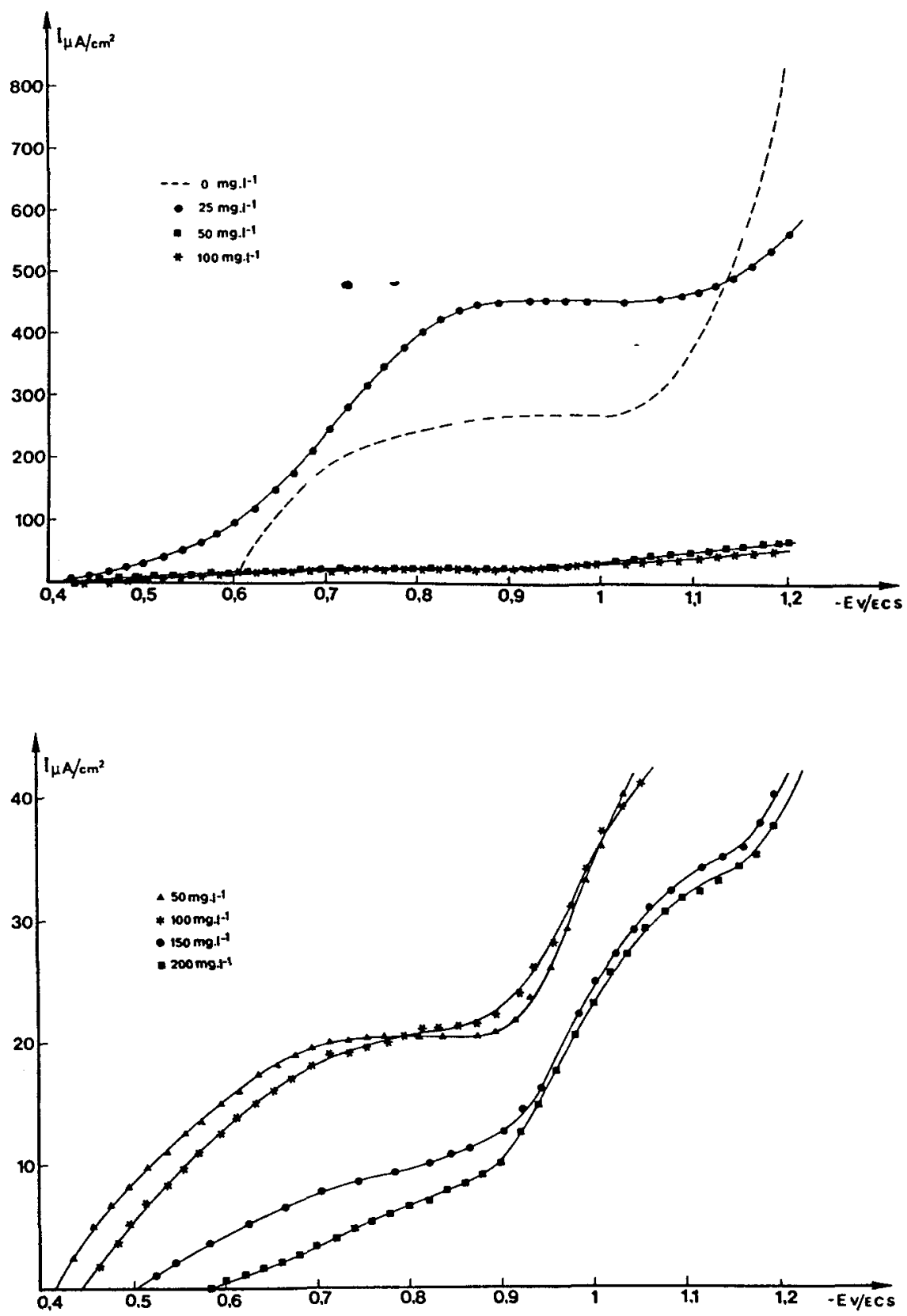

Figures 1 et 2 Courbes courant-tension cathodiques relevées en mode potentiostatique pour différentes concentrations en inhibiteur.

Interface acier XC $35 / \mathrm{NaC̣I} 200 \mathrm{mg} \cdot 1^{-1}$.

Cathodic current-voltage curves (potentiostatic regulation) plotted for different inhibitor concentrations. 
Tableau 1 Paramètres obtenus à partir des courbes courant-tension cathodiques pour différentes concentrations en inhibiteur.

Interface acier XC 35/ $\mathrm{NaCl} 200 \mathrm{mg} \cdot 1^{-1}$.

Table 1 Parameters deduced from the cathodic current-voltage curves for different inhibitor concentrations.

\begin{tabular}{|ccccc|}
\hline $\begin{array}{c}\text { Concentration } \\
\text { on inhibiteur } \\
\left(\mathbf{m g} \cdot \mathbf{H}^{-1}\right)\end{array}$ & $\begin{array}{c}\mathbf{E}_{\text {corr }} \\
(\mathbf{m V} / \mathrm{ECS})\end{array}$ & $\begin{array}{c}\text { Ilimite } \\
\left(\mu \mathbf{A} / \mathbf{c m}^{2}\right)\end{array}$ & $\begin{array}{c}\mathrm{I}_{\text {corr }} \\
\left(\mu \mathbf{A} / \mathrm{cm}^{2}\right)\end{array}$ & $\begin{array}{c}\mathbf{E} \\
(\%)\end{array}$ \\
\hline 0 & $-685 \pm 5$ & $250 \pm 20$ & $100 \pm 10$ & - \\
25 & $-414 \pm 4$ & $525 \pm 75$ & $19 \pm 1$ & 81 \\
50 & $-422 \pm 2$ & $20 \pm 0$ & $4,5 \pm 0,5$ & 95 \\
100 & $-437 \pm 3$ & $23 \pm 2$ & $4,5 \pm 0,5$ & 95 \\
150 & $-520 \pm 10$ & $13 \pm 0$ & $3 \pm 1$ & 97 \\
200 & $-585 \pm 5$ & $11 \pm 0$ & $2,3 \pm 0,5$ & 98 \\
\hline
\end{tabular}

\section{b) Dans le domaine anodique}

Nous avons tracé les courbes anodiques $E=f(I)$ en mode potentiostatique, en l'absence et en présence de l'inhibiteur. Pour ce type d'essai, nous avons retenu la concentration de $100 \mathrm{mg} \cdot 1^{-1}$ pour laquelle il a été montré une importante efficacité inhibitrice dans le domaine cathodique.

L'étude comparée des courbes stationnaires anodiques (fig. 3) en présence et en l'absence de l'inhibiteur montre que l'adjonction du composé au milieu entraîne une augmentation de la surtension pour une densité de courant donnée, au voisinage de $E_{\text {corr. }}$ Pour de fortes densités de courant $\left(I>500 \mu \mathrm{A} \cdot \mathrm{cm}^{-2}\right)$, il n'y a pas de différence notable entre les courbes avec et sans inhibiteur. La densité de courant de corrosion, obtenue en l'absence d'inhibiteur, par extrapolation au potentiel de corrosion, de la droite de Tafel (150 $\pm 40 \mu \mathrm{A} \cdot \mathrm{cm}^{-2}$ ) voit sa valeur fortement abaissée en présence de $100 \mathrm{mg} \cdot 1^{-1}$ d'inhibiteur $\left(.2 \mu \mathrm{A} \cdot \mathrm{cm}^{-2}\right)$. Ces résultats sont en accord avec ceux obtenus dans le domaine cathodique. De plus, la surface de l'électrode, après 5 heures d'immersion, ne présente aucune trace de corrosion; ceci plaide en faveur d'une action anodique importante de l'inhibiteur, vraisemblablement liée à la présence de phosphonate.

\subsubsection{Mesures d'impédance électrochimique}

Les diagrammes d'impédance déterminés au potentiel de corrosion pour différentes concentrations en inhibiteur sont donnés sur la (fig. 4). Ils se caractérisent par une seule boucle capacitive fortement déformée dont la taille croît lorsque la concentration augmente. A partir de $100 \mathrm{mg} \cdot 1^{-1}$, le diagramme se caractérise par une partie linéaire dans le domaine des hautes fréquences. Un tel comportement a déjà été observé par DUPRAT, SRHIRI, DERBALI et PEBERE (1986), lors de l'inhibition de la corrosion d'un acier au carbone par des monoalkyiphosphonates. II est caractéristique de l'existence d'une relaxation du transport de matière. Ainsi, la boucle capacitive observée n'est pas représentative d'une seule constante de temps mais est plutôt le reflet de la contribution du transfert de charge et du transport de matière. Cette observation justifie le choix d'appeler $R_{p}$ la résistance pour $\omega \rightarrow 0$ et non $R_{T}$ (résistance de transfert de charge). 


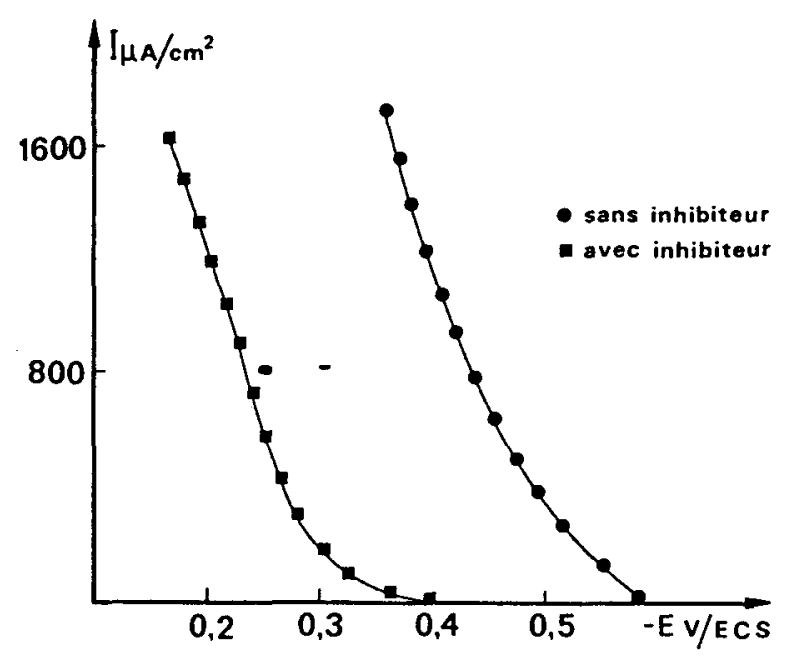

Figure 3 Courbes courant-tension anodiques relevées en mode potentiostatique en l'absence et en présence de $100 \mathrm{mg} \cdot 1^{-1}$ d'inhibiteur.

Anodic current-voltage curves (potentiostatic regulation) without and with inhibitor $(100 \mathrm{mg} \cdot+1)$.

Le comportement capacitif de l'interface devient de plus en plus marqué lorsque la concentration augmente.

Pour les concentrations de 150 et $200 \mathrm{mg} \cdot 1^{-1}$, la détermination de la résistance de polarisation devient délicate et avoisinerait des valeurs de l'ordre de 20000 à $30000 \Omega \cdot \mathrm{cm}^{2}$.

On notera que les valeurs de la résistance de polarisation, obtenues pour les différentes concentrations et mesurées à partir des diagrammes d'impédance sont en accord avec la pente locale des courbes courant-tension au voisinage de $\mathrm{E}_{\text {corr }}$.

De plus, on constate une bonne corrélation entre les valeurs de la résistance de polarisation, $R_{p}$, et le pourcentage de protection observé.

II convient de souligner que l'aplatissement des diagrammes observé en l'absence et en présence de l'inhibiteur, semble être en partie due à la faible conductivité du milieu étudié.

\subsection{Influence du pH}

Les courbes courant-tension cathodiques et les diagrammes d'impédance électrochimique ont été relevés d'une part, dans la solution à $200 \mathrm{mg} \cdot \mathrm{I}^{-1} \mathrm{de}$ chlorure de sodium et, d'autre part, dans cette même solution en présence de $100 \mathrm{mg} \cdot \mathrm{l}^{-1}$ d'inhibiteur, pour des valeurs de $\mathrm{pH}$ de 5,5-7-8 et 9 . 

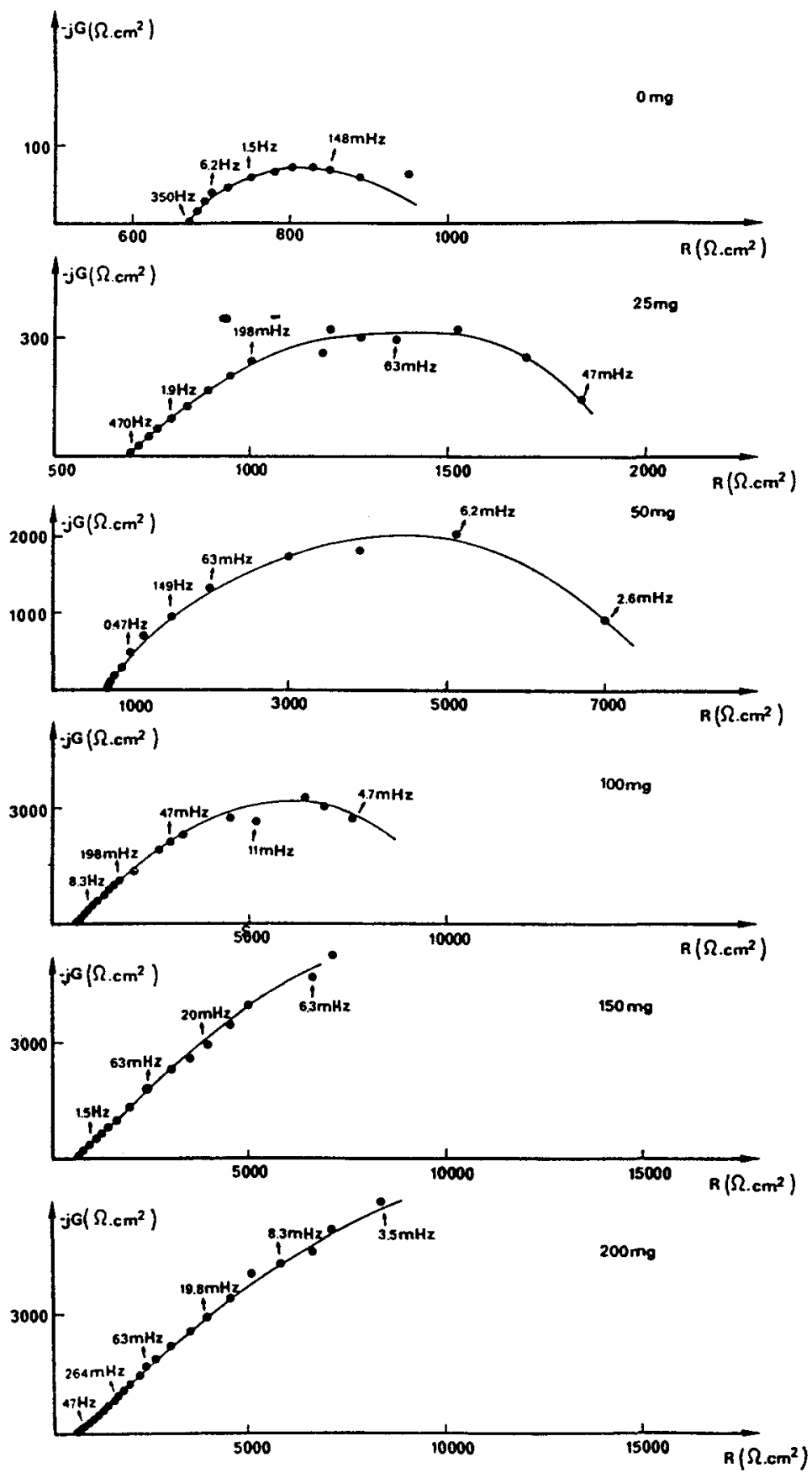

Figure 4 Diagrammes d'impédance électrochimique relevés au potentiel de corrosion pour différentes concentrations en inhibiteur $(\mathrm{pH}=7)$.

Electrochemical impedance diagrams corresponding to potentiostatic regulation for different inhibitor concentrations $(\mathrm{pH}=7)$. 


\subsubsection{Courbes courant-tension cathodiques}

Les figures 5 et 6 représentent les courbes de polarisation tracées respectivement en l'absence et en présence de l'inhibiteur pour différentes valeurs du $\mathrm{pH}$ de la solution.

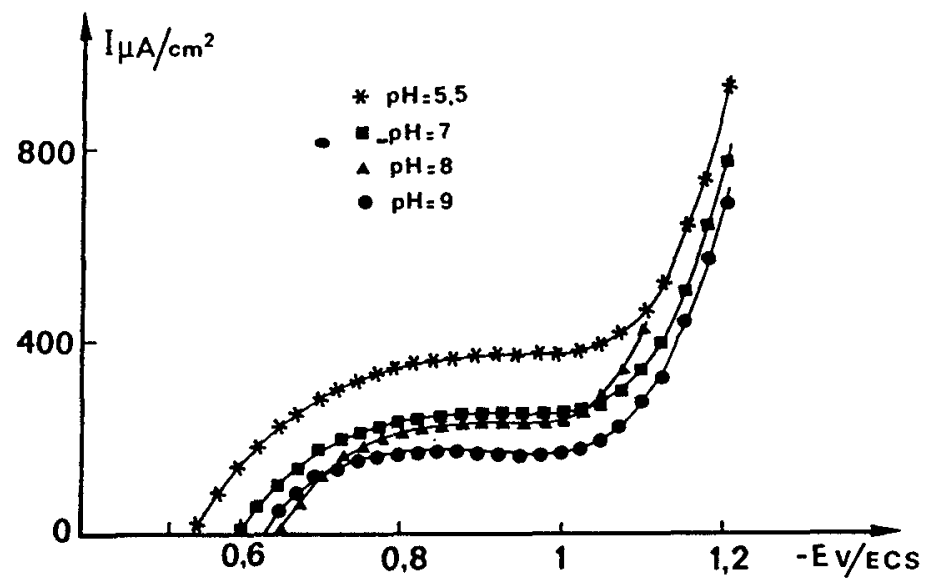

Figure 5 Courbes courant-tension cathodiques relevées en mode potentiostatique pour différentes valeurs du pH de la solution agressive, en l'absence d'inhibiteur.

Interface acier XC 35/ $\mathrm{NaCl} 200 \mathrm{mg} \cdot \mathrm{I}^{-1}$.

Cathodic current-voltage curves (potentiostatic regulation) for different $\mathrm{pH}$ values of the corrosive solution - without inhibitor.

En l'absence d'inhibiteur, on note un déplacement du potentiel de corrosion vers des potentiels plus cathodiques ainsi qu'une diminution de la hauteur du palier de diffusion avec l'augmentation du $\mathrm{pH}$. Cette différence entre les densités de courant limite de diffusion peut s'expliquer par un effet « barrière de diffusion " créé par l'accumulation de produits de corrosion à la surface de l'électrode. En effet, ces produits de corrosion sont de plus en plus denses et moins solubles lorsque le pH augmente.

Les densités de courant de corrosion ont été déterminées par extrapolation au potentiel de corrosion de la droite de TAFEL corrigée de la diffusion sauf, dans le cas du $\mathrm{pH}=9$ où le courant de corrosion a été pris égal au courant limite de diffusion. Les vitesses de corrosion ainsi mesurées traduisent une corrosion plus importante à pH $=7$ qu'à $\mathrm{pH}=5,5$ (tableau 2) et nettement plus forte à $\mathrm{pH}=8$ et à $\mathrm{pH}=9$.

Ce résultat est en contradiction avec des travaux antérieurs réalisés en milieu chlorure de sodium à $3 \%$ par DUPRAT et DABOSI (1981) qui montraient une faible dépendance de la vitesse de corrosion dans ce domaine de $\mathrm{pH}$. Cependant, l'observation de l'état de surface à la fin de nos essais électrochimiques révèle une couche importante de produits de corrosion avec sous celle-ci une attaque profonde lorsque le $\mathrm{pH}$ augmente, cet examen confirmerait une augmentation de la corrosion avec le $\mathrm{pH}$. 


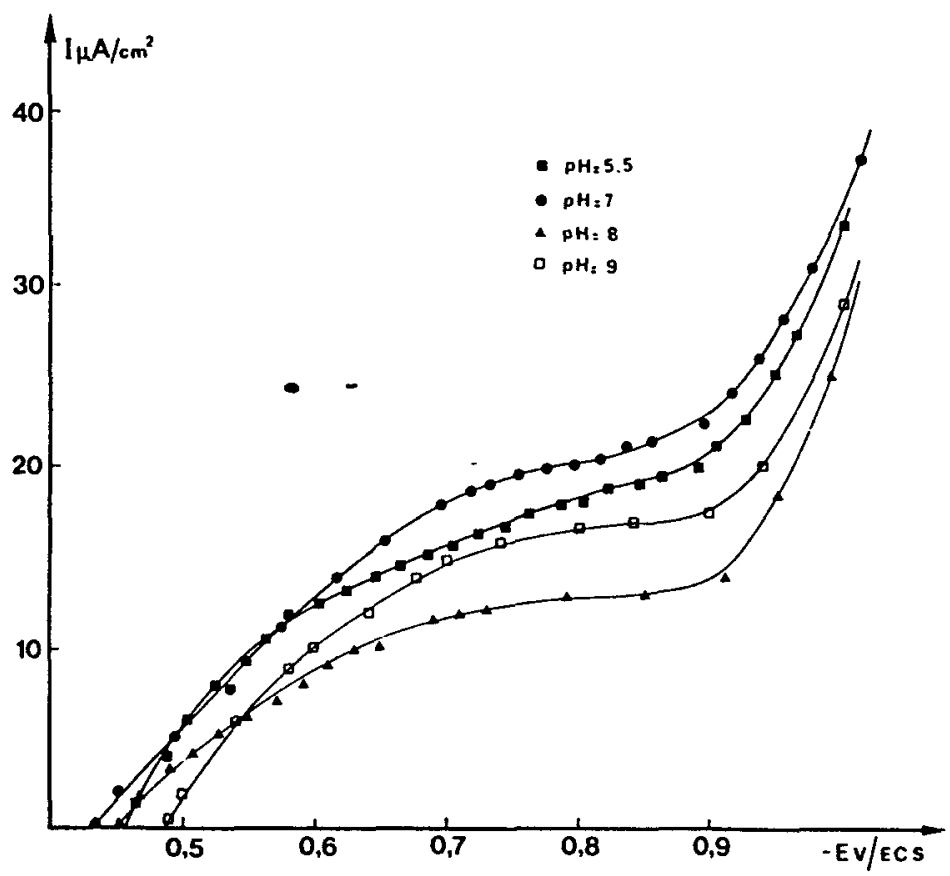

Figure 6 Idem figure 5 en présence de $100 \mathrm{mg} \cdot 1^{-1}$ d'inhibiteur. Idem figure 5 with inhibitor $\left(100 \mathrm{mg} \cdot r^{-1}\right)$.

Tableau 2 Paramètres obtenus à partir des courbes courant-tension cathodiques pour différentes valeurs de $\mathrm{pH}$, en l'absence d'inhibiteur. Interface acier XC $35 / \mathrm{NaCl} 200 \mathrm{mg} \cdot 1^{-1}$.

Table 2 Parameters deduced from the cathodic current-voltage curves for different $\mathrm{pH}$ values without inhibitor.

\begin{tabular}{|c|c|c|c|}
\hline pH & $\begin{array}{c}E_{\operatorname{cor}} \\
(m V / E C S)\end{array}$ & $\begin{array}{c}\text { limite } \\
\left(\mu \mathrm{A} / \mathrm{cm}^{2}\right)\end{array}$ & $\begin{array}{c}I_{\text {corr }} \\
(\mu \mathrm{A} / \mathrm{cm} 2)\end{array}$ \\
\hline $\begin{array}{l}5,5 \\
7 \\
8 \\
9\end{array}$ & $\begin{array}{l}-510 \pm 10 \\
-585 \pm 5 \\
-650 \pm 10 \\
-625 \pm 5\end{array}$ & $\begin{array}{l}390 \pm 10 \\
250 \pm 20 \\
225 \pm 25 \\
195 \pm 10\end{array}$ & $\begin{array}{c}75 \pm 5 \\
100 \\
175 \pm 5 \\
195 \pm 10\end{array}$ \\
\hline
\end{tabular}

La présence de l'inhibiteur diminue de façon importante les densités de courant (fig. 6) par rapport au milieu non inhibé, conduisant à une diminution significative de la vitesse de corrosion (tableau 3). Ce résultat montre donc la très bonne efficacité de l'inhibiteur dans le domaine de $\mathrm{pH}$ étudié et plus particulièrement pour le $\mathrm{pH}$ de 8 . Toutefois, à $\mathrm{pH}=5,5$, l'efficacité mesurée n'est que de $91 \%$ et quelques traces de corrosion ont pu être observées. 
Tableau 3 Paramètres caractéristiques obtenus à partir des courbes de polarisation cathodique pour différentes valeurs de $\mathrm{pH}$, avec $100 \mathrm{mg} \cdot \mathrm{I}^{-1}$ d'inhibiteur.

Table 3 Parameters deduced from the cathodic current-voltage curves for different $\mathrm{pH}$ values $\left(100 \mathrm{mg} \cdot \mathrm{H}^{-1}\right)$.

\begin{tabular}{|c|c|c|c|c|}
\hline $\mathrm{pH}$ & $\begin{array}{c}E_{\mathrm{cor}} \\
(\mathrm{mV} / \mathrm{ECS})\end{array}$ & $\begin{array}{c}\text { limilo } \\
\left(\mu \mathrm{A} / \mathrm{cm}^{2}\right)\end{array}$ & $\begin{array}{c}I_{\text {corr }} \\
(\mu A / c m 2)\end{array}$ & $\begin{array}{c}E \\
(\%)\end{array}$ \\
\hline $\begin{array}{l}5,5 \\
7 \\
8 \\
9\end{array}$ & $\begin{array}{c}-460 \\
-437 \pm 3 \\
-450 \pm 10 \\
-470 \pm 20\end{array}$ & $\begin{array}{c}20 \\
23 \pm 2 \\
12 \pm 1 \\
25 \pm 5\end{array}$ & $\begin{array}{c}7 \\
4,5 \pm 0,5 \\
2 \pm 0,5 \\
5 \pm 1\end{array}$ & $\begin{array}{l}91 \\
95 \\
99 \\
97\end{array}$ \\
\hline
\end{tabular}

\subsubsection{Mesures d 'impédance électrochimique}

La figure 7 présente les diagrammes d'impédance obtenus avec $100 \mathrm{mg} \cdot \mathrm{I}^{-1}$ d'inhibiteur pour trois valeurs du $\mathrm{pH}$. Les valeurs des résistances de polarisation mesurées par extrapolation de la partie basse fréquence du diagramme de 10000 à $20000 \Omega \cdot \mathrm{cm}^{2}$ confirment les résultats obtenus à partir des courbes stationnaires: une très bonne efficacité du composé en particulier à $\mathrm{pH}=8$.

\section{3 Étude comparative de l'efficacité inhibitrice de quelques composés utilisés dans le traitement de l'eau}

Sur la figure 8, nous avons comparé les efficacités inhibitrices du chlorure de zinc, du monofluorophosphate de zinc et de la formulation testée ici, en fonction de leur concentration ramenée à la concentration molaire de zinc.

Cette figure révèle que la vitesse de corrosion, $i_{\text {corr }}$ apparaît très faible dans le cas de l'addition des produits dérivés de phosphonates en association avec des sels de zinc et qu'elle diminue progressivement lorsque la concentration en inhibiteur augmente.

Pour le chlorure de zinc, l'efficacité inhibitrice obtenue n'est que de $80 \%$, tandis que pour le monofluorophosphate de zinc, la vitesse de corrosion ne diminue pas de façon monotone avec la concentration d'inhibiteur. En effet, on observe une valeur minimale de $i_{\text {corr }}\left(11 \mu \mathrm{A} \cdot \mathrm{cm}^{-2}\right)$ pour une concentration de $6 \times 10^{-5} \mathrm{M}$. Pour des concentrations supérieures de $\mathrm{ZnPO}_{3} \mathrm{~F}, \mathrm{i}_{\text {corr }}$ croît. Un tel phénomène a déjà été mis en évidence par LAHODNY-SARC et KARTELAN (1976) - notamment dans le cas des polyphosphates - et peut trouver son origine dans le fait qu'aux fortes concentrations, il se formerait un complexe métallique soluble qui entraînerait une accélération de la corrosion. Dans toute la gamme de concentration étudiée le monofluorophosphate de zinc est moins efficace que les produits dérivés de phosphonates en association avec les sels de zinc.

En outre, si l'on compare ces résultats à une autre formulation inhibitrice testée précédemment par DUPRAT, LAFONT, MORAN et ROCHER (1985), composée de l'association d'une amine grasse et d'acide phosphonique, les vitesses 
de corrosion apparaissent plus faibles pour les phosphonates associés aux sels de zinc. En effet, pour $\left.1 \mathrm{~g} \cdot\right|^{-1}$ du mélange d'amine grasse et d'acide phosphonique, la densité de courant de corrosion mesurée est de $20 \mu \mathrm{A} \cdot \mathrm{cm}^{-2}$, pour les produits dérivés de phosphonates en association avec des sels de zinc, les vitesses de corrosion varient de 5 à $2 \mu \mathrm{A} \cdot \mathrm{cm}^{-2}$ en fonction de la concentration en inhibiteur.
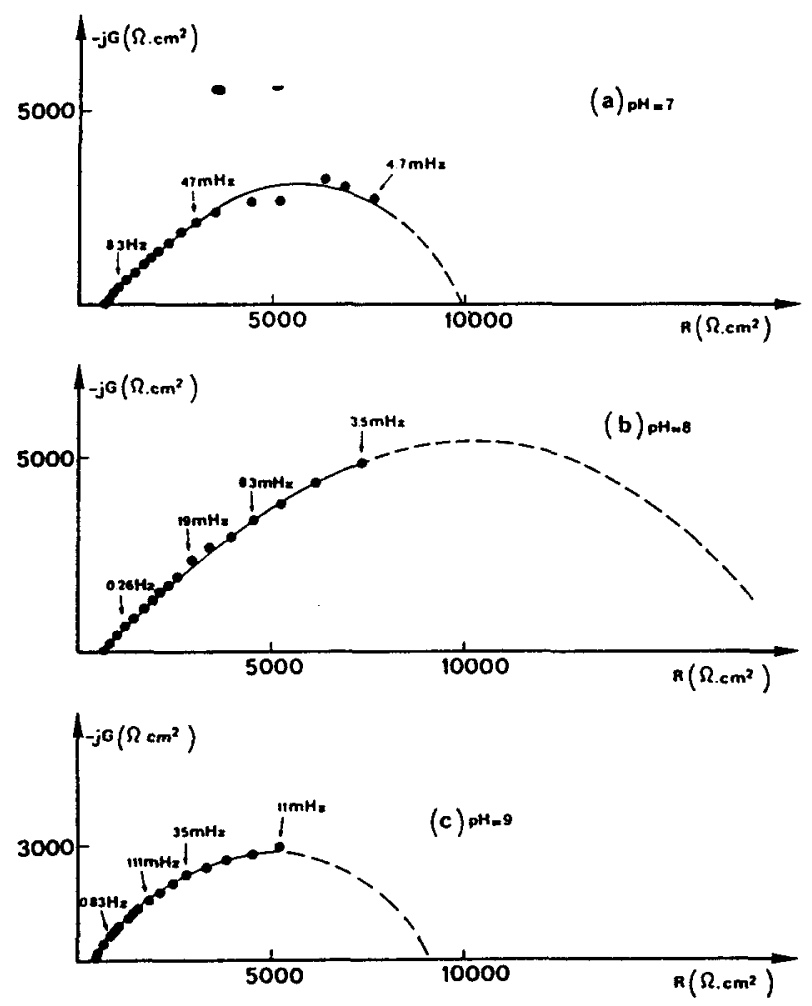

Figure 7 Diagrammes d'impédance électrochimique relevés au potentiel de corrosion pour différentes valeur du $\mathrm{pH}$ de la solution.

Interface acier XC $35 / \mathrm{NaCl} 200 \mathrm{mg} \cdot 1^{-1}+100 \mathrm{mg} \cdot 1^{-1}$ d'inhibiteur.

Electrochemical impedance diagrams corresponding to potentiostatic regulation for different $\mathrm{pH}$ values with inhibitor $\left(100 \mathrm{mg} \cdot \mathrm{t}^{-1}\right)$.

\section{4 - CONCLUSION}

Cette étude montre l'intérêt de l'utilisation des produits dérivés de phosphonates associés aux sels de zinc comme inhibiteur de corrosion pour le traitement des eaux industrielles. 


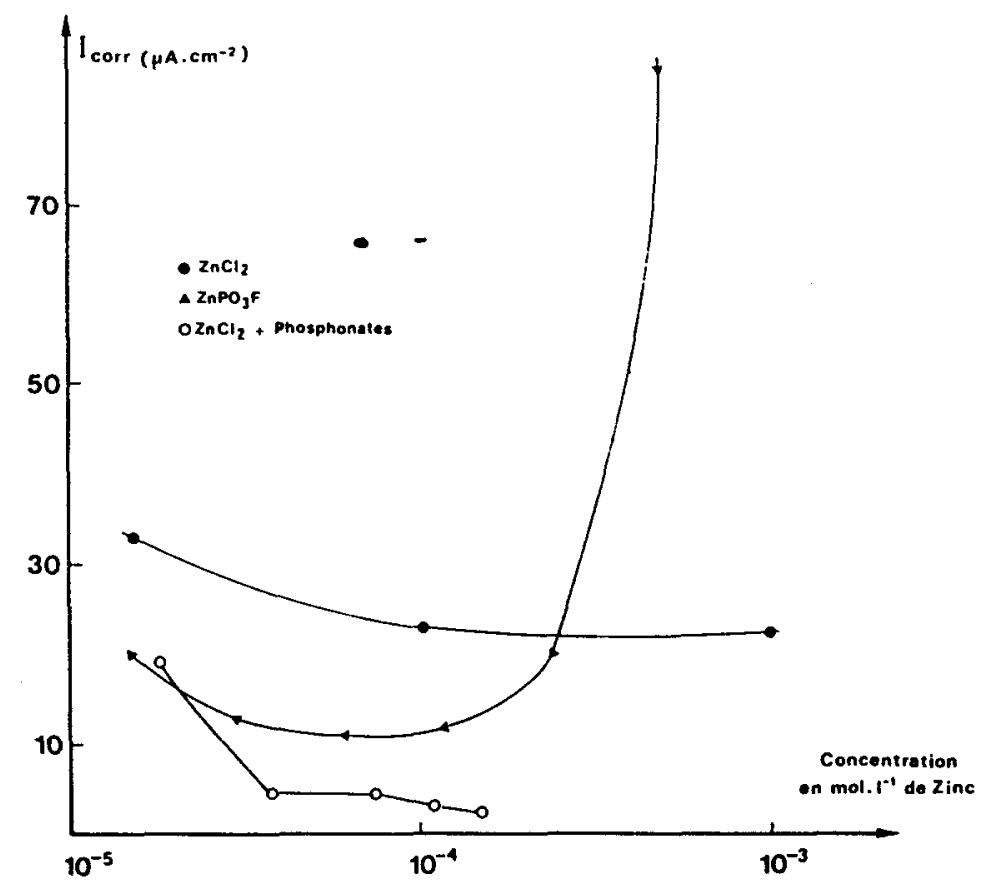

Figure 8 Variation de la vitesse de corrosion en fonction de la concentration molaire de zinc pour différents inhibiteurs.

Corrosion rate versus zinc concentration for different compounds.

Les essais électrochimiques ont montré la très bonne efficacité du composé pour les faibles concentrations (dès $50 \mathrm{mg} \cdot \mathrm{r}^{-1}$ ). Pour la concentration de $100 \mathrm{mg} \cdot \mathrm{F}^{-1}$, il est efficace dans un domaine de $\mathrm{pH}$ relativement large (de 5,5 à 9). Cette efficacité apparaît légèrement supérieure à $\mathrm{pH}=7$ et à $\mathrm{pH}=8$.

Le produit testé agit comme un inhibiteur mixte. Cette observation est importante dans l'hypothèse de l'utilisation d'une quantité insuffisante de produit.

L'étude comparative de l'efficacité inhibitrice du chlorure de zinc, du monofluorophosphate de zinc, d'un mélange d'une amine grasse et d'acide phosphonique et de l'association phosphonates/sel de zinc fait apparaître une meilleure efficacité de cette dernière quel que soit le domaine de concentration étudié.

Une étude est actuellement en cours dans le but d'obtenir une meilleure connaissance des mécanismes mis en jeu lors du processus d'inhibition du mélange étudié. 


\section{RÉFÉRENCES BIBLIOGRAPHIQUES}

DUPRAT M., DABOSI F., 1981. Corrosion inhibition of a carbon steel in $3 \% \mathrm{NaCl}$ solutions by aliphatic Amino-Alcohol and Diamine type compounds. Corrosion, 37 : 89-92.

DUPRAT, M., LAFONT, M.C., MORAN, F., ROCHER, S., 1985. Study of the corrosion and inhibition processes of a carbon steel in a low conductivity medium by electrochemical methods. Electrochimica Acta, 30 (3) : 353-365.

DUPRAT, M., LAFONT, M.C., MORAN, F., ROCHER, S., 1985. Inhibition de la corrosion d'un acier au carbone en milieux neutres aérés par les monofluorophos- phates. Revue Française des Sciences de rEau, 4 : 1-15.

DUPRAT, M., LAFONT, M.C., MORAN, F., ROCHER, S., 1985. Une méthode nouvelle d'inhibition de la corrosion due aux eaux industrielles. L'Eau. I'Industrie. les Nuisances, $89:$ 41-44.

DUPRAT, M., SRHIRI, A., DERBALI, Y., PEBERE, N., 1986. An electrochemical impedance approach to the corrosion inhibition of a carbon steel in neutral media. Materials Science Forum, $8: 267-280$.

LAHODNY-SARC O., KARTELAN, L., 1976. Inhibition of mild steel by polyphosphates. Corrosion Science, $16: 25-34$.

\section{Le Culde aux auteurs est disponible aux adresses suivantes:}

\section{REVUE DES SCIENCES DE L'EAU}

Jean-Pierre Villeneuve

INRS-Eau

Université du Québec 2800, rue Einstein

CP 7500

SAINTE-FOY (Qué)

Canada G1V 4C7

Tél. : (418) 654-2592

Fax : (418) 654-2660
André Rambaud

Département Sciences

de l'Environnement

et Santé Publique

Faculté de Pharmacie

Avenue Charles Flahault 34060 MONTPELLIER Cedex

Tél. : 67630109

Fax : 67045933 